\title{
The yeast MUD2 protein: an interaction with PRP11 defines a bridge between commitment complexes and U2 snRNP addition
}

\author{
Nadja Abovich, ${ }^{1}$ Xiaoling C. Liao, ${ }^{1,2}$ and Michael Rosbash ${ }^{1,3}$ \\ ${ }^{1}$ Howard Hughes Medical Institute, Brandeis University, Department of Biology, Waltham, Massachusetts 02254 USA
}

\begin{abstract}
In characterizing a series of yeast (Saccharomyces cerevisiae) mutants synthetic lethal with U1 RNA, we have identified a yeast gene (MUD2) with sequence similarity to the well-studied metazoan splicing factor U2AF65. The biochemical characterization indicates that the MUD2 gene product (MUD2P) contacts pre-mRNA directly and is a component of the pre-mRNA-U1 snRNP complex (commitment complex) that forms during early spliceosome assembly in yeast extracts. Unlike U1 snRNP itself, the association of MUD2P with pre-mRNA is dependent on a proper yeast branchpoint sequence. Genetic experiments indicate that MUD2P affects U2 snRNP addition. Moreover, experiments in the two-hybrid system show that PRP11P, a recently identified component of $U 2$ snRNP, can interact directly with MUD2P. The experiments identify a specific inter-snRNP protein-protein contact that occurs during spliceosome assembly and more generally support substantial functional similarity between U2AF65 and MUD2P.
\end{abstract}

[Key Words: Yeast; pre-mRNA splicing; snRNP]

Received January 5, 1994; revised version accepted February 22, 1994.

The field of pre-mRNA splicing has adopted the working hypothesis that the efficiency and specificity of the cleavage and ligation reactions are provided by the five splicing small ribonucleoprotein particles (snRNPs) (Guthrie 1991; Steitz 1992; Moore et al. 1993). This chemical role is preceded by an ordered snRNP addition pathway that occurs during in vitro spliceosome assembly. U1 snRNP is the first snRNP to interact with the pre-mRNA substrate. This is followed by the ATP-dependent binding of $U 2$ snRNP, which is followed in turn by the binding of the U4/U5/U6 tri-snRNP (for reviews, see Green 1991; Rymond and Rosbash 1992; Moore et al. 1993). Although it is not known whether this pathway also occurs in vivo, its conservation between two wellstudied in vitro splicing systems, yeast (Saccharomyces cerevisiae) and mammals, suggests that it is of functional significance.

Studies in both systems indicated that the binding of U1 snRNP is important to form a stable complex (a "commitment complex"), committed to the rest of the splicing pathway (Séraphin and Rosbash 1989; Michaud and Reed 1991, 1993; Jamison et al. 1992). Although U1 snRNP binding may be aided and perhaps even preceded by the association of important protein factors with the

${ }^{2}$ Present address: Department of Microbiology and Immunology, University of California, San Francisco, California 94143 USA.

${ }^{3}$ Corresponding author.
pre-mRNA substrate (Zamore and Green 1989; Bennett et al. 1992; Michaud and Reed 1993; Fu 1993; Wu and Maniatis 1993), there are a number of in vitro observations indicating that U1 snRNP interacts with both the $5^{\prime}$ and $3^{\prime}$ splice site regions during the early phases of splicing complex formation (Legrain et al. 1988; Ruby and Abelson 1988; Zillmann et al. 1988; Barabino et al. 1990; Séraphin and Rosbash 1991; Hoffman and Grabowski 1992; Jamison et al. 1992; Michaud and Reed 1993).

The $5^{\prime}$ splice site region is a short sequence quite conserved between yeast and mammals /consensus: G-GUAUGU in yeast and G-GUAAGU in mammals where the dash indicates the $5^{\prime}$ splice site). The best characterized aspect of its interaction with U1 snRNP involves direct base-pairing with the $5^{\prime}$ end of Ul RNA (for review, see Rosbash and Séraphin 1991). The 3' splice site region is more complex and consists of two subregions: (1) the conserved dinucleotide at the 3 ' splice site (AG) and a usually adjacent polypyrimidine-rich stretch, and (2) a nearby branchpoint sequence. In mammalian systems, the interaction between the $3^{\prime}$ splice site region and U1 snRNP depends in part on indirect contacts between the pre-mRNA polypyrimidine stretch and U1 snRNP (Hoffman and Grabowski 1992; Michaud and Reed 1993; Wu and Maniatis 1993), whereas the highly conserved branchpoint sequence (UACUAAC) appears to collaborate with the $5^{\prime}$ splice site region to pro- 
mote U1 snRNP complex formation in the yeast $S$. cerevisiae (Séraphin and Rosbash 1989). The relative importance of the branchpoint subregion or the rest of the $3^{\prime}$ splice site region to yeast and mammalian complex formation, respectively, reflects their conservation among different pre-mRNAs: The polypyrimidine stretch is a less prominent feature of many yeast pre-mRNAs (although, see Patterson and Guthrie 1991), whereas the branchpoint sequence (highly conserved as UACUAAC in yeast; $\mathbf{A}$ indicates branchpoint) is much more variable among mammalian pre-mRNAs (Rymond and Rosbash 1992). The relative importance of these two subregions is also reflected in their contribution to the first step of splicing in yeast: In this system, a truncated pre-mRNA substrate can undergo spliceosome assembly and the first step of splicing quite efficiently without a prominent polypyrimidine stretch and a $3^{\prime}$ splice site AG (e.g., Rymond and Rosbash 1985).

The contribution of the branchpoint region to yeast U1 snRNP complex formation in vitro was detected by electrophoresis assays, which showed that wild-type substrates form two ATP-independent commitment complexes, CC1 and CC2 (Séraphin and Rosbash 1989). Substrates with mutant or missing branchpoint regions only form the higher mobility complex (CC1) (Séraphin and Rosbash 1991/. A number of observations indicate that $\mathrm{CC} 2$ corresponds to a larger more complete complex and that some of the factors in this complex do not associate or associate less stably with U1 snRNP and premRNA in the absence of a functional branchpoint region (Séraphin and Rosbash 1991). This reasoning suggests that proteins present in $\mathrm{CC} 2$, but absent in $\mathrm{CCl}$, might depend on, and perhaps associate with, the branchpoint region during this early step in spliceosome assembly.

Previously, we had undertaken a genetic approach to identify U1 snRNP proteins or protein factors that collaborate with U1 snRNP during pre-mRNA splicing (Liao et al. 1993). This enhancer or synthetic lethal screen searched for mutant genes that were nonfunctional in combination with mutant versions of U1 RNA but functional in a wild-type background. We employed a biochemical subscreen to define several candidate genes that also affected U1 snRNP complex formation in vitro. The first of these genes characterized in detail (MUD1) encodes the S. cerevisiae version of the wellstudied mammalian U1 snRNP protein U1A (Liao et al. 1993).

To identify candidate proteins involved in branchpoint recognition during commitment complex formation, we screened mutant extracts for specific defects in $\mathrm{CC} 2$ formation. One gene (MUD2) fulfilled this criterion and was studied in detail. As expected from the initial characterization of the mutant extract, it associates directly with the pre-mRNA substrate in a branchpoint-dependent fashion. Remarkably, it encodes a protein related to the well-studied splicing factor U2AF65 (Ruskin et al. 1988; Zamore et al. 1992), which also interacts with the $3^{\prime}$ splice site region during early complex formation in mammalian systems (Zamore and Green 1989; Bennett et al. 1992; Michaud and Reed 1993). The results support previously suspected similarities as well as differences in the early events of spliceosome formation between yeast and mammals. They also provide insight into possible functional roles of U2AF during these early steps of complex formation.

\section{Results}

One mutant (mud2) fulfilled the criterion for further study: Extracts from the four alleles that were tested were able to form CC1, the basal pre-mRNA-Ul snRNP complexes, but little or no $\mathrm{CC} 2$, the complete premRNA-U1 snRNP complex (data not shown). Because these in vitro phenotypes were based on a synthetic lethal combination (the mud mutant in addition to a significant deletion in UI RNA) and because there might be more than one relevant mutation in the mutagenized background, we cloned the MUD2 gene and generated a gene deletion (MUD2 KO; see Materials and methods). Like the original mud2 mutants, the deletion had no detectable growth phenotype in an otherwise wild-type background. We then constructed an epitope-tagged version of MUD2 by adding the 9-amino-acid-epitope from influenza virus hemagglutinin protein (HA) to the carboxyl terminus of MUD2P. The tagged gene (MUD2 HA) functioned indistinguishably from the wild-type $M U D 2$ gene, as it rescued the synthetic lethality of the mud2U1 RNA deletion combination (data not shown). Extracts were prepared from these strains and commitment complex formation assayed by native gel electrophoresis (Fig. 1).

The results showed that in the MUD2 KO extracts, the CC2 complex migrated more rapidly than the same complex formed in a wild-type extract (cf. lane 5 with lane 1). As expected from the phenotypic rescue, the extract from the MUD2 HA strain formed a normal (wild-typelike) CC2 complex (lane 3). Addition of the 12CA5 monoclonal antibody to the extracts prior to electrophoresis caused a supershift of only CC2 from the MUD2 HA extract (lane 4), indicating that MUD2P is itself a component of the $\mathrm{CC} 2$ complex rather than having an indirect effect on complex migration. The specificity of the supershift was verified by adding the same antibody to an extract derived from a strain in which the U170K protein had been similarly tagged with the HA antibody (U170K HA). In this case (lane 2), both $\mathrm{CCl}$ and CC2 were supershifted, consistent with the previous finding that Ul snRNP is a component of both commitment complexes.

The association of MUD2 with U1 snRNP was assayed further by immunoprecipitation of U1 RNA from MUD2 HA extracts with the 12CA5 antibody (Fig. 2A). In all cases, little U1 snRNP was immunoprecipitated as compared with the positive control, the U170K HA extract (cf. lanes 9-12 with lanes 5-8). This indicates that unlike the U170K protein, MUD2 is not stably associated with U1 snRNP in extracts. As pre-mRNA addition increased the amount of immunoprecipitated U1 RNA from MUD2 HA extracts, the stable association of MUD2 with U1 snRNP appeared pre-mRNA dependent (cf. lane 


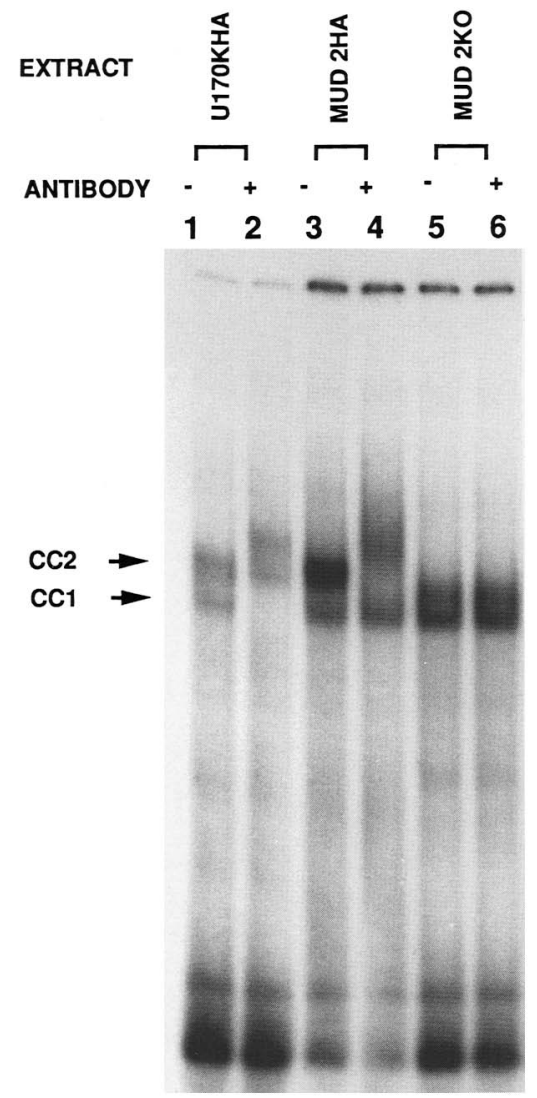

Figure 1. Native gel electrophoresis of commitment complexes. Splicing extracts prepared from the indicated strains were incubated with radioactive wild-type pre-mRNA $\Delta 2-3$ and an oligonucleotide complementary to U2 RNA to form commitment complexes as described previously (Liao et al. 1992). Prior to electrophoresis, 12CA5 monoclonal antibody was added to the reactions in lanes 2,4 , and 6 to supershift the complexes containing the HA-tagged proteins as described in Materials and methods. Although the amount of total commitment complex is variable among different extracts, there was no consistent difference in the ratio of $\mathrm{CC} 1 / \mathrm{CC} 2$ between MUD2 HA and wild-type extracts. The two forms of the commitment complex are indicated.

10 with lane 9|. Pre-mRNA substrate specificity was tested by comparing RNAs with mutations in the two important regions for early spliceosome assembly steps, the $5^{\prime}$ splice site and the branchpoint regions. The $5^{\prime}$ splice site mutant contains a substitution in the universally conserved $G$ at position 5 of the $5^{\prime}$ splice site (GUAUGU $\rightarrow$ GUAUaU), which has a significant effect on in vitro splicing and commitment complex formation. Consistent with this observation, there was less U1 snRNP immunoprecipitation with the mutant substrate (cf. lane 11 with lane 10). A deletion of the 7-nucleotide branchpoint region reduced the association to background levels (cf. lane 12 with lane 9). The fact that MUD2P-U1 snRNA association is dependent on a proper pre-mRNA branchpoint sequence is consistent with the assignment of the MUD2-U1 snRNP associa- tion to CC2, whose formation is similarly dependent on the branchpoint region (Séraphin and Rosbash 1991).

The association of MUD2P with pre-mRNA was directly assayed by immunoprecipitation with the 12CA5 antibody (Fig. 2B). From two different MUD2-tagged extracts, substantial levels of pre-mRNA were recovered, well above the background levels observed from a wildtype extract (cf. lanes 3 and 4 with lane 1). Yet the premRNA levels from the MUD2 HA extracts were only $30-50 \%$ of those from the U170K HA extract, consistent with the notion that only the CC2 subset of U1 snRNPpre-mRNA complexes contains the MUD2 protein. With the mutant 5' splice site, pre-mRNA immunoprecipitation was reduced substantially, but the relative amount of pre-mRNA precipitated in the three HA-tagged extracts was unaffected by the mutation, consistent with the failure of this mutation to substantially alter the CC1/CC2 ratio (Séraphin and Rosbash 1991). A qualitatively different result was obtained with a 7 nucleotide deletion of the branchpoint sequence: There was little effect on immunoprecipitation from the U170K HA strain but a dramatic decrease in immunoprecipitation from the MUD2 HA extracts. In these cases, pre-mRNA levels were not appreciably greater than background /cf. lanes 11 and 12 with lane 9), consistent with the assignment of MUD2 to the branchpoint-dependent CC2 complex.

Overexpression of MUD2HAP, by expression from a galactose upstream activating sequence (UAS)-containing expression construct, had no detectable effect on the level of pre-mRNA immunoprecipitation (e.g., Fig. 2B, cf. lane 4 with lane 3 ). This is not attributable to the lack of an effect on protein levels, because the MUD2 HA Gal extract had at least 10 times as much MUD2P as the MUD2 HAYCP extract (assayed by Western blotting with the 12CA5 antibody; data not shown). The negative result suggests that MUD2P levels are not limiting in wild-type extracts and that the protein interacts only weakly with pre-mRNA in the absence of commitment complex formation.

The association of MUD2 with pre-mRNA was assayed further by UV cross-linking. Subsequent to irradiation, reactions were digested with RNase A, denatured, and immunoprecipitated with 12CA5 antibody from MUD2 HA extracts or from U170K HA extracts (Fig. 3). The results with a wild-type substrate indicate that MUD2 is in direct contact with the pre-mRNA. The results with mutant substrates are consistent with those shown above: The $5^{\prime}$ splice site mutation reduced the signal and a strong branchpoint mutation (UACUACC) eliminated the signal. All of the in vitro data indicate that MUD2P interacts directly with the pre-mRNA substrate in a branchpoint-dependent fashion.

The sequence of $M U D 2$ indicated that it codes for a protein of $58 \mathrm{kD}$. The four sequenced mud2 alleles introduced stop codons within this open reading frame (Materials and methods). Although no strong homologies were apparent in data base searches, a few known RNAbinding proteins were among the best matches. These proteins were identified because of the ribonucleopro- 

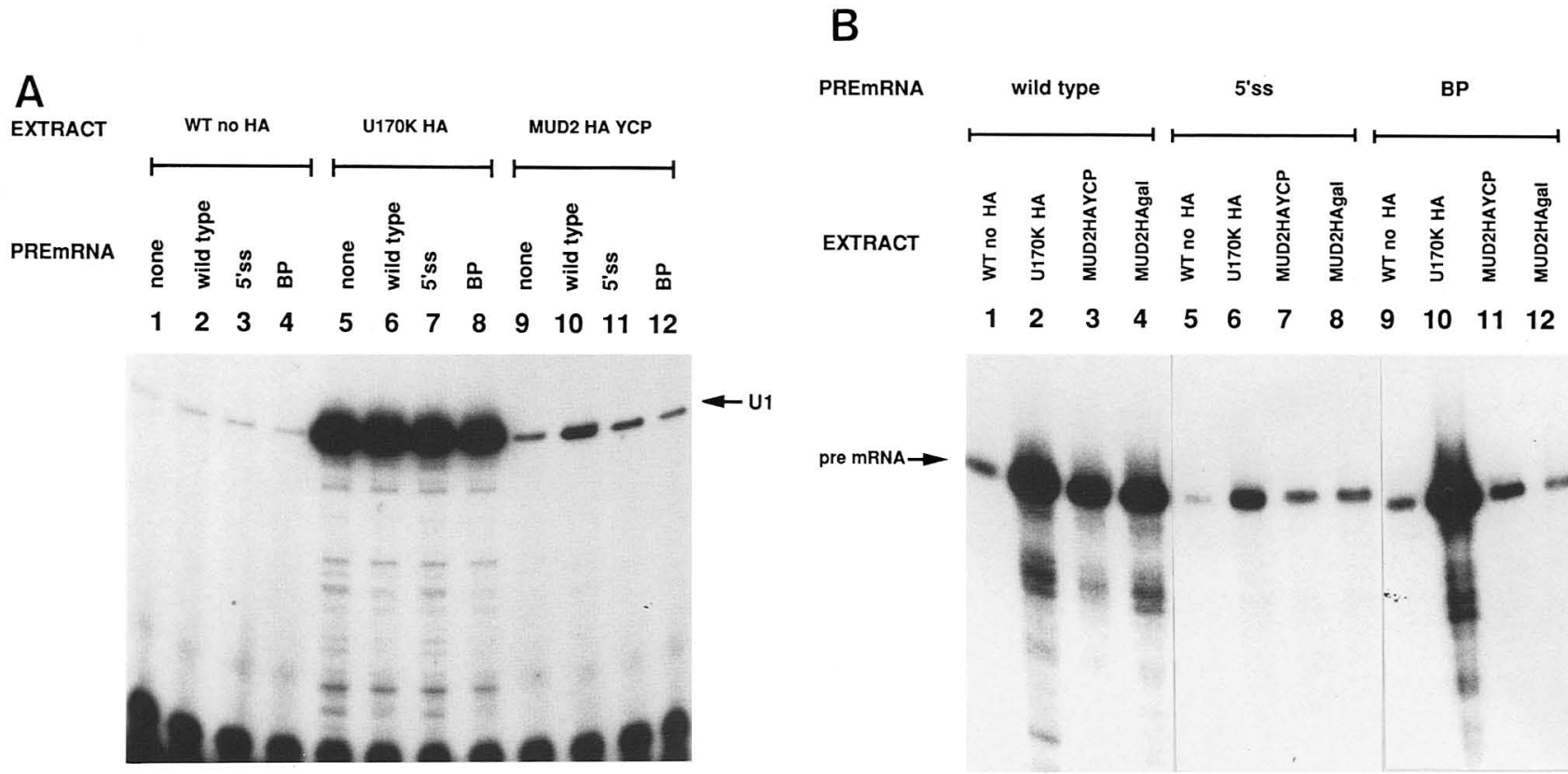

Figure 2. Associations of MUD2P. (A) Association with UI RNA. Commitment complexes were formed as in Fig. 1 with the indicated extracts; no pre-mRNA was added to the reactions in lanes 1,5, and 9, or $25 \mathrm{ng}$ of nonradiolabeled pre-mRNAs as indicated in each lane. The $5^{\prime}$ splice site (5'ss) pre-mRNA contained the sequence GUAUaU, and the BP pre-mRNA contained a 7-nucleotide deletion of the branchpoint sequence UACUAAC (Séraphin and Rosbash 1991). After immunoprecipitation and washing in NET-150, the RNA was recovered and cDNA was synthesized with reverse transcriptase and a 5 '-labeled oligonucleotide specific for U1 RNA. (B) Association with pre-mRNA. Complexes were formed as above and an extract was prepared from a strain carrying the MUD2 HA-tagged protein under the control of the Gal promoter in a multicopy vector (lanes $4,8,12$ ). Radiolabeled pre-mRNAs were added as indicated above. After immunoprecipitation under the same conditions described above, these pre-mRNAs were recovered and electrophoresed on denaturing urea-acrylamide gels.

tein consensus sequence (RNP-CS) or RNA-binding domain (RBD) present in MUD2, and they included a single known splicing factor, U2AF65, with a molecular weight similar to MUD2.

U2AF65 has been shown to interact directly with the polypyrimidine-rich portion of the mammalian $3^{\prime}$ splice site region prior to U2 snRNP addition (Ruskin et al. 1988; Zamore and Green 1989). It is an essential factor for mammalian and Drosophila in vitro splicing (Zamore et al. 1992; Kanaar et al. 1993); cognates in Drosophila (Kanaar et al. 1993) and Schizosaccharomyces pombe (fission yeast; Potashkin et al. 1993) have recently been identified, and it is an essential gene in these two organisms. Because the interaction of MUD2P with yeast premRNA also takes place prior to U2 snRNP addition and is dependent on a proper branchpoint region, this relationship was examined in more detail.

A multisequence alignment of MUD2P with the three U2AF65 genes is shown in Figure 4A. Each metazoan U2AF65 has an arginine-serine (RS)-rich domain at its amino terminus followed by three RBDs. Even the $S$. pombe sequence shows $50 \%$ overall sequence conservation and a similar organization with an RS domain followed by three RBDs. In contrast, MUD2P contains only a single easily identifiable RBD near its carboxyl terminus, but its closest relative in the data base is the car- boxy-terminal RBD of hU2AF65 (31\% identity and 49\% similarity|.

The rest of U2AF65 is not convincingly related to MUD2P, nor does MUD2P encode additional recognizable RBDs. However, there are some hints that the proteins might share features in addition to this single RBD. First, there is an intriguing patch of homology with U2AF65 (especially with the human and Drosophila versions) near the end of the first RBD region, comprising amino acids 303-311 (Fig. 4). Second, the amino-terminal region of MUD2 is rich in arginine and contains four RS (or SR) dipeptides. Although this does not constitute a striking RS domain like the comparable region of hU2AF65, the dU2AF65 protein only contains three RS (or SR) dipeptides in its amino-terminal domain. Third, there is a similar distribution of histidines and cysteines throughout the hU2AF and MUD2 proteins, with the histidines clustered at the amino terminus and the cysteines at the carboxyl terminus, further suggesting that some aspects of functional conservation might extend beyond the carboxy-terminal RBDs. In sum, the two proteins are related, but the similarity is insufficient to identify MUD2P as S. cerevisiae's U2AF65 protein.

To verify the functional importance of the conserved carboxy-terminal RBD, we mutagenized the two highly conserved aromatic residues present in the RNP-1 sub- 


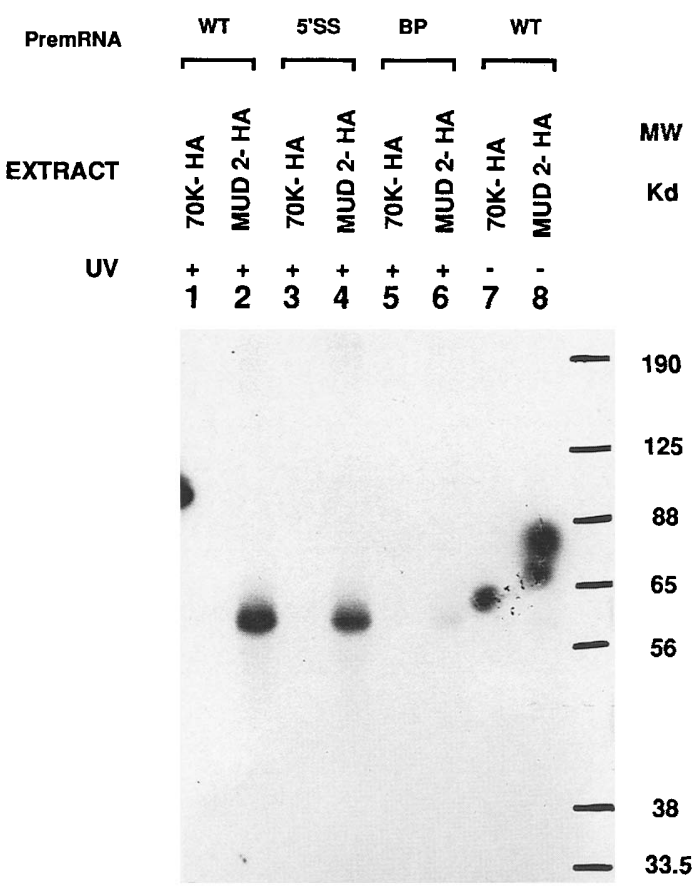

Figure 3. UV cross-link of MUD2 protein to pre-mRNA. Splicing reactions for commitment complex were scaled up sixfold, with the U170K or MUD2-tagged extracts and the indicated radiolabeled pre-mRNAs. The complexes were UV irradiated (lanes 1-6) and RNase A treated (see Materials and methods). Prior to immunoprecipitation, the samples were denatured in SDS as described and diluted back to NET-150. Immunoprecipitates were washed at NET-300, and SDS sample buffer was added directly to the beads and boiled. The proteins were electrophoresed on a 10\% SDS-PAGE gel (Laemmli 1970) and the UV cross-linked species detected by autoradiography. The position of prestained molecular mass markers is indicated at right. The signals in the negative control lanes $(7,8)$ do not correspond to radioactive proteins.

region of this region. This subregion in general and these amino acids in particular have been shown to be important for the RNA-binding function of RBDs. The bioassay determined the ability of the mutant proteins to rescue the synthetic lethality of a $M U D 1-M U D 2$ double KO (see below), by replacing the wild-type MUD2 gene with the mud2 mutant genes. Although the first aromatic residue was inessential, the mutant protein with both residues changed to aspartic acid was unable to rescue the synthetic lethality of the MUD1-MUD2 double KO combination (Fig. 5). Similar results have been reported for the mutagenesis of the RNP1 subregion of another RBD-containing mammalian protein, the splicing factor ASF/SF2 (Caceres and Krainer 1993; Zuo and Manley 1993). The double mutation also eliminated in vitro activity, as extracts that expressed this mutant MUD2 protein (indistinguishably from the wild-type protein by Western blotting with the 12CA5 antibody; data not shown/ were identical to the negative control MUD2 KO strain. (The in vitro role was defined by UV cross-linking to pre-mRNA as well as by native gel electrophoresis; data not shown). The results indicate that this region of MUD2 contributes to its splicing function.

Because of the importance of U2AF for U2 snRNP binding, we sought to determine whether MUD2P might play an analogous (albeit inessential) role during $S$. cerevisiae splicing. This was first assayed genetically, by placing the MUD2 KO in combination with other prp mutants known to be components of U2 snRNP or to affect U2 snRNP activity (Fig. 6). One of these temperature-sensitive mutants, prp11 (Chang et al. 1988), normally grows well at $25^{\circ} \mathrm{C}$ but failed to grow at this temperature in combination with the MUD2 KO gene. The synthetic lethality was not attributable to some general difficulty of MUD2KO-prp mutant combinations: The growth of two other prp temperature-sensitive mutants, another U2 snRNP temperature-sensitive mutation (prp9, Abovich et al. 1990) and a U5 snRNP temperaturesensitive mutation (prp8, Lossky et al. 1987) was unaffected by the addition of the MUD2 KO allele. As a positive control, the MUD2 KO was also put in combination with a $M U D 13 \mathrm{KO}$ [a recently characterized inessential U1 snRNP protein (H. Colot, unpubl.)] as well as with the MUD1 KO; consistent with the notion that all three proteins contribute to a single activity (U1 snRNP|, both combinations were synthetic lethal.

Because the human PRP11 protein (SAP62) (Bennett and Reed 1993) is a subunit of SF3A, which is a loosely associated component of U2 snRNP in mammalian systems (Brosi et al. 1993), the synthetic lethality suggested that MUD2 is a commitment complex component that affects $\mathrm{U} 2$ snRNP addition. An alternative interpretation is based on recent work suggesting that, in yeast, PRP11 adds to U1 snRNP complexes in an ATP-independent manner, for example, prior to U2 snRNP addition (Ruby et al. 1993). These latter observations resemble those made for MUD2 and more generally for CC2 formation, suggesting that the mud2-prp11 synthetic lethality might reflect their contribution to the same particle or assembly step.

To distinguish between these possibilities, we assayed the U2 RNA dependence and ATP dependence of PRP11 addition to pre-mRNA (and Ul snRNP) with procedures similar to those used above for MUD2. Extracts were made from a prp11 temperature-sensitive strain rescued with a tagged PRP11 protein, which was expressed from a promoter containing a galactose UAS (see Materials and methods). Although a low level of pre-mRNA was immunoprecipitated in the absence of U2 RNA or ATP, this amount was dramatically increased in their presence (Fig. 7A). Similarly, PRP11's association with U1 RNA is dependent on functional U2 RNA (Fig. 7B) as well as ATP (data not shown). The low levels of immunoprecipitated U1 RNA and pre-mRNA are likely attributable to the trace amounts of U2 RNA refractory to RNase $\mathrm{H}$ digestion (Fig. 7B cf. lanes 2 and 3, and 6 and 7, with 1 and 5, respectively). The results are identical to those obtained for PRP9 (Abovich et al. 1990) and PRP21 (Arenas and Abelson 1993), at least one of which (PRP9) is also a known component of SF3A. Taken together, the 
Abovich et al.

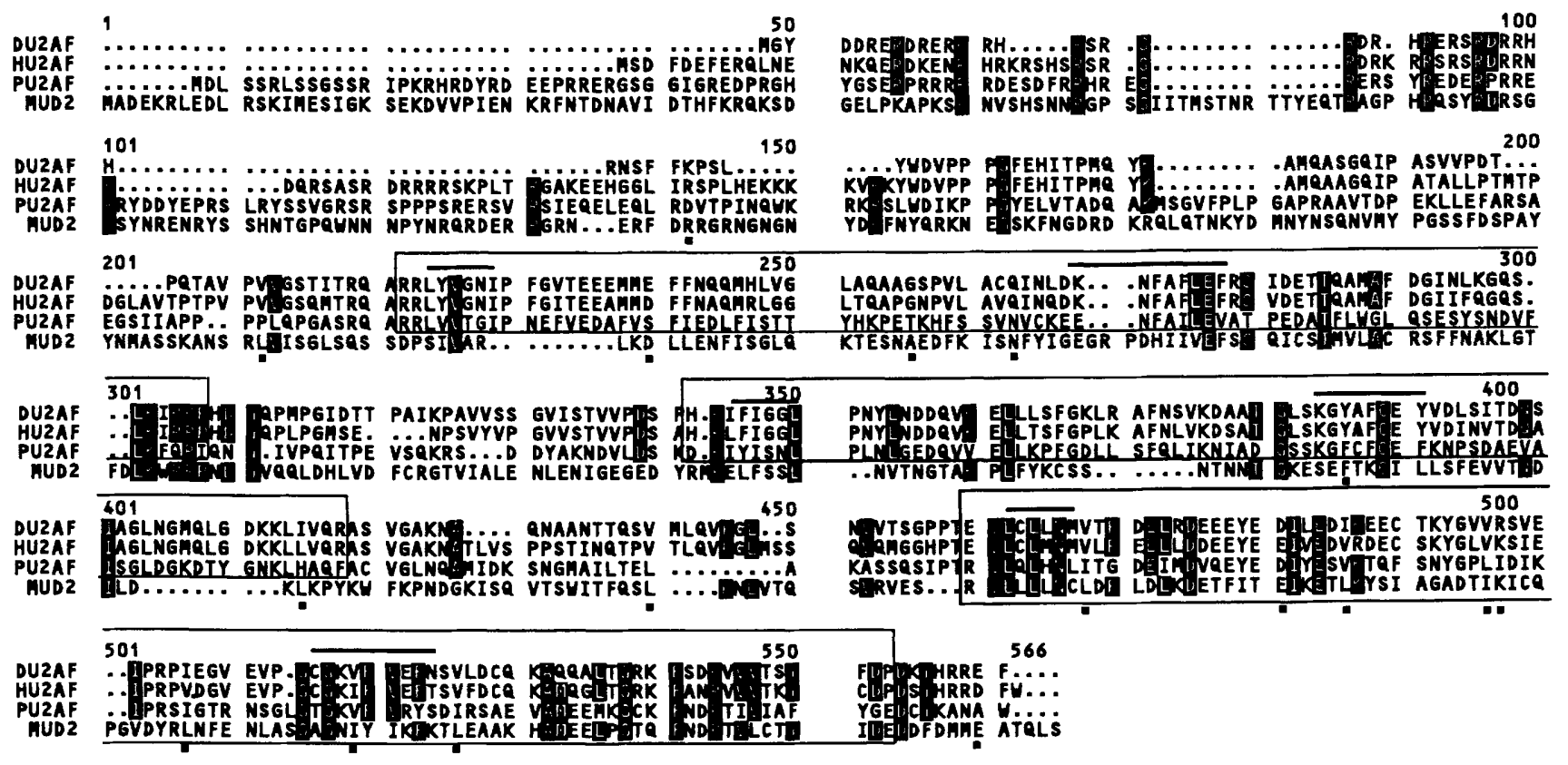

Figure 4. Alignment of MUD2, Drosophila (DU2AF ${ }^{50}$, Kanaar et al. 1993), human (HU2AF ${ }^{65}$, Zamore et al. 1992) and S. pombe PRP2 (PU2AF, Potashkin et al. 1993) proteins using the program Pileup, with a gap weight of 3.0 and gap length weight of 0.1 . The three U2AF sequences were manually moved two spaces to align with MUD2 at position 303 . The three RBDs are boxed in and within each the RNP-1 and RNP-2 conserved elements are overlined (Kanaar et al. 1993). The shaded areas represent positions of identity in all four proteins: Three identical and one conservative change or three identical amino acids, one of which is on MUD2. The small black boxes represent positions at which at least three positions are conserved, one of them being MUD2. Similar amino acids were considered $T$ and $\mathrm{S} ; \mathrm{V}, \mathrm{L}$ and $\mathrm{I} ; \mathrm{D}$ and $\mathrm{E} ; \mathrm{K}$ and $\mathrm{R} ; \mathrm{N}$ and $\mathrm{Q} ; \mathrm{G}$ and $\mathrm{A} ; \mathrm{F}$ and $\mathrm{Y}$.

results indicate that PRP1l adds to U1 snRNP complexes as a component of U2 snRNP.

This interpretation then raises the question, does MUD2 affect U2 snRNP addition directly, for example, does MUD2 make physical contact with U2 snRNP and in particular with PRP11? We assayed the physical association of MUD2 and PRP11 in the yeast two-hybrid system, where a positive result was obtained (Fig. 8). Posi- tive and negative controls consisted of pieces of the Drosophila period gene, known to undergo homodimerization in vitro (Huang et al. 1993) as well as in this yeast assay (Z. Huang, unpubl.). The prpll temperature-sensitive mutant protein as well as PRP11-Cys (in which the two cysteines of the zinc finger were altered) interacted with MUD2P indistinguishably from PRP11P (Fig. 8), and the $\mathrm{YF} \rightarrow \mathrm{DD}$ change in the RBD of MUD2P was

\section{STRAIN}

MUD1 KO (ADE) / MUD2 KO (LEU) + MUD1 (URA) YCP.

+MUD2 HA (TRP)
+VECTOR (TRP)
+MUD2 HA Y=D (TRP)
+MUD2 HA YF=DD (TRP)

\section{FOA LEU-}

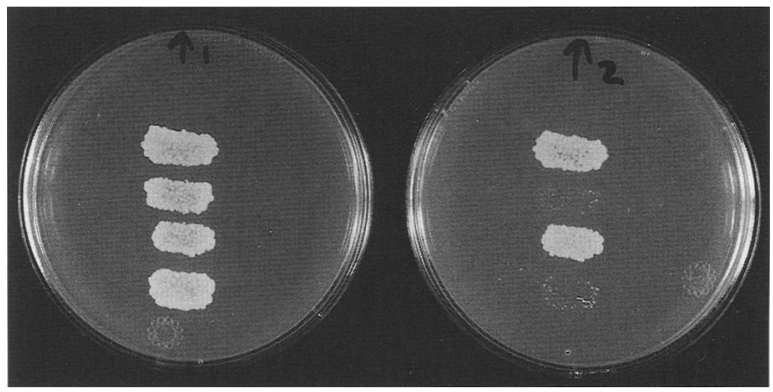

Figure 5. Effect of RNP-1 mutations on the activity of MUD2P. A strain was constructed in which both genes MUD2 and MUD1 had been deleted by replacement of the coding regions with the LEU2 and ADE2 genes, respectively, (see Materials and methods; Liao et al. 1993). The strain also carries the MUD1 gene in a centromeric plasmid YCP50 containing the URA3 gene, for a positive selection of cells that can lose the plasmid and grow on 5-fluoro-orotic acid (FOA). Mutations in the RNP-1 region of MUD2 Y $\rightarrow$ D and $\mathrm{YF} \rightarrow \mathrm{DD}$ were introduced in HA-tagged MUD2P by oligonucleotide-directed mutagenesis (Kunkel 1985) and transferred to the PG1 vector (Schena and Yamamoto 1988) for transformation and TRP selection. Transformants were grown on minimal plates selected for adenine, leucine, and tryptophan and replica plated to FOA Leu- ${ }^{-}$plates. 


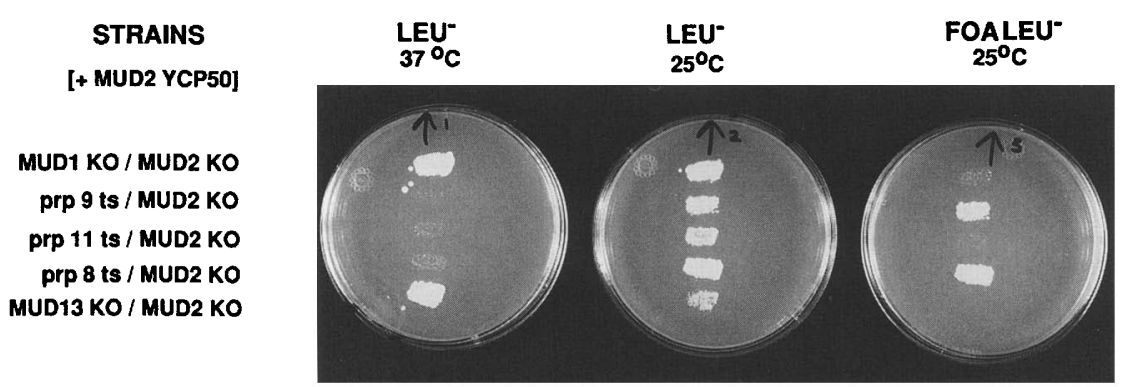

Figure 6. Synthetic lethal relationships of MUD2. The MUD2 gene was deleted in each of the strains and replaced by the LEU2 gene. The deletion was covered by the wild-type $M U D 2$ gene in the URA3containing centromeric plasmid YCP50. The strains were patched on uracil and leucine selective plates and maintained at $25^{\circ} \mathrm{C}$. They were replica plated onto the indicated selective plates. similarly without effect (data not shown). Although an association of two yeast proteins in this assay must be interpreted with caution, the results are consistent with the in vivo synthetic lethality and suggest that MUD2P and PRP11P are part of the interface between U1 and U2 snRNP during spliceosome formation.

\section{Discussion}

Previous experiments in this laboratory were interpreted to indicate that the difference in mobility between the two U1 snRNP complexes formed with a wild-type substrate was attributable to a component $(X)$ that associ- ates with pre-mRNA in a branchpoint dependent fashion (Séraphin and Rosbash 1991) (Fig. 9A). This report defines MUD2P as a specific subcomponent of $\mathrm{X}$ and the first identified protein factor component of commitment complexes (Fig. 9B). The conclusion is based on biochemical criteria as well as the synthetic lethal interaction of mud2 with U1 RNA and two bona fide U1 snRNP proteins.

Although the data indicate that there is a tripartite association among pre-mRNA, Ul snRNP, and $X$ for MUD2 as a component of $X \mid$, they do not give insight into order of addition considerations, that is, does MUD2P addition precede, accompany, or follow U1 sn-

A
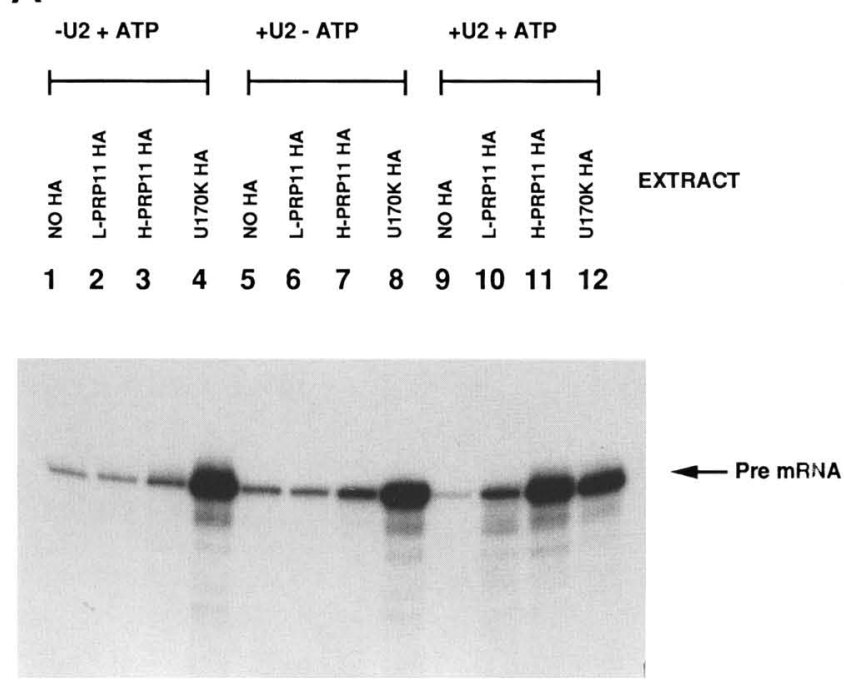

B
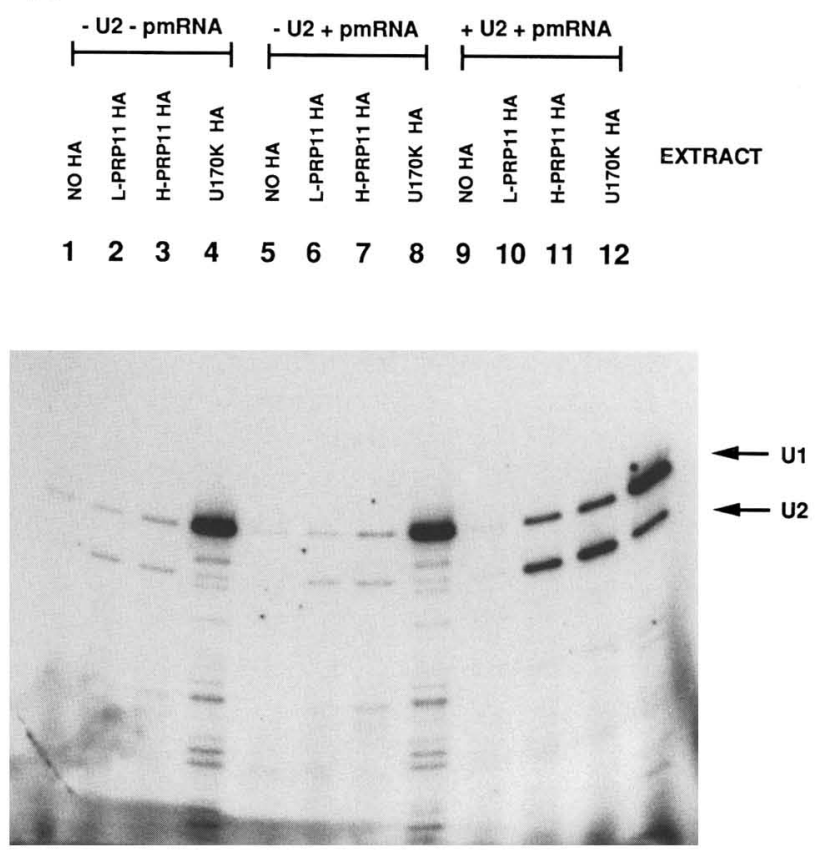

Figure 7. Immunoprecipitations in a PRP11-HA-tagged extract. $(A)$ pre-mRNA. Radiolabeled wild-type pre-mRNA was added to splicing reactions in extracts prepared from a wild-type strain (lanes $1,5,9$; the prpl1 temperature-sensitive strain rescued with an HA-tagged PRP11 gene under the galactose promoter whose expression was repressed in glucose-containing media (lanes 2,6,10, L-PRP11HA) or induced in galactose-containing media (lanes 3,7,11, H-PRP11HA); or the U170K HA strain (lanes 4,8,12). The reactions contained an oligonucleotide complementary to U2 RNA to effectively deplete U2 RNA activities (-U2) (lanes 1-4) or 2 mM glucose to deplete the endogenous ATP in the extracts (lanes 4-8). (B) U1 and U2 snRNPs. The reactions were as $A$ except that nonradiolabeled pre-mRNA (25 ng) was added to the reactions in lanes 5-12. After immunoprecipitation, the recovered RNAs were used as templates for reverse transcription from $5^{\prime}$-labeled oligonucleotides complementary to U1 and U2 RNAs. The cDNA products are indicated. 
Figure 8. In vivo interaction between MUD2 and PRP11 proteins. Yeast cells EGY 48 carrying a reporter gene, LexA op-Gall-LacZ (pSH 18-34), were transformed with plasmids containing LexA fusion proteins under a constitutive promoter and with plasmids in which the indicated proteins were fused to the Escherichia coli activating sequence B42. These latter plasmids are expressed from a galactose-inducible promoter. MUD2 and PRP11 fusions contained the complete protein; in prp11 the prp11-1 temperaturesensitive protein has been fused to $\mathrm{B} 42$ and in PRP11-Cys the cysteine residues at 68 and 71 have been changed to glycines. The Drosophila period fusions comprise amino acids 233-685 (Huang et al. 1993).

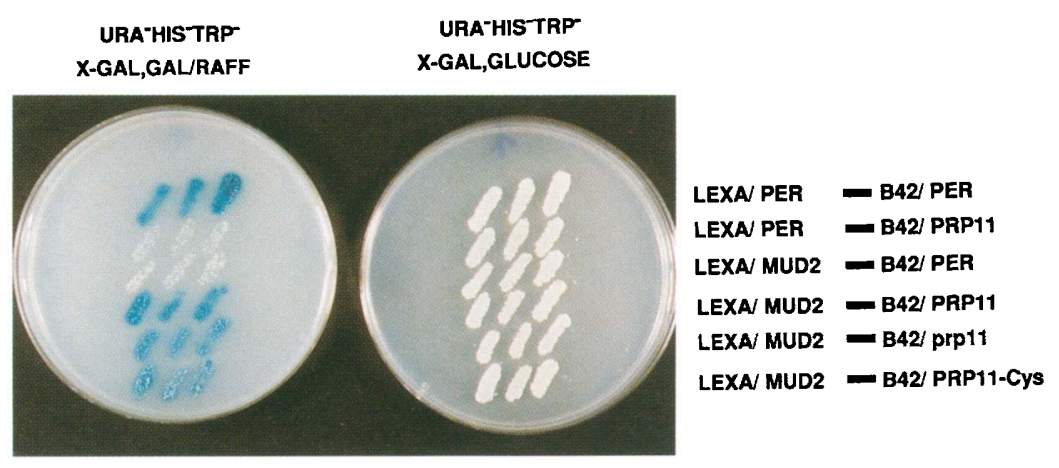

RNP-pre-mRNA complex formation? This problem parallels our lack of understanding of the relationship between $\mathrm{CCl}$ and $\mathrm{CC} 2$. These might have a precursorproduct relationship. Alternatively, $\mathrm{CCl}$ might be an in vitro artifact caused by the dissociation of $X$, a phenomenon that might be substantially enhanced in the absence of a pre-mRNA branchpoint region (Séraphin and Rosbash 1991). We favor this second interpretation, because we have never been able to verify a precursorproduct relationship (Liao et al. 1992). Moreover, a direct examination of commitment complex salt sensitivity indicates that $\mathrm{CC} 2$ converts into $\mathrm{CC} 1$ upon exposure to increasing salt concentrations (H.V. Colot, unpubl.); the association of MUD2P with both pre-mRNA and U1 snRNP is similarly salt sensitive (data not shown).

Although the 3' splice site region is important to early complex formation in both yeast and mammals, several lines of evidence indicate that only the highly conserved branchpoint subregion contributes substantially to early complex formation in yeast. In mammals, the branchpoint subregion is poorly conserved and the 3 ' splice site AG and (usually) adjacent polypyrimidine-rich subregions appear more important for early events (for reviews, see Green 1991; Rymond and Rosbash 1992; Moore et al. 1993). It is possible that the interaction of U2AF65 with the polypyrimidine track and the interaction of $X$ with the yeast branchpoint region play similar roles, namely, to stabilize a factor that associates with U1 snRNP and then helps recruit U2 snRNP to the 3' splice site region. A role for U2AF in U2 snRNP recruitment is well established (Ruskin et al. 1988; Zamore and Green 1991), and there are several lines of evidence that U2AF65 also interacts with U1 snRNP (Bennett et al. 1992; Hoffman and Grabowski 1992; Michaud and Reed 1993). A very recent study describes a series of interactions that bridge U2AF65 and U1 snRNP $70 \mathrm{~K}$ protein (Wu and Maniatis 1993).

More generally, is MUD2P the U2AF65 of S. cerevisiae? U2AF65 is essential both in Drosophila (Kanaar et al. 1993) and S. pombe (Potashkin et al. 1993), allowing the possibility of another essential $S$. cerevisiae gene product more closely related to U2AF65 than MUD2P. Yet, the highly conserved U1 snRNP A protein is an inessential splicing protein in S. cerevisiae (Liao et al. 1993), making the interpretation "inessential" not straightforward. Also, the MUD2KO strain has a twofold effect on the splicing of the inefficient reporter gene construct Acc as compared with an isogenic wild-type strain (Liao et al. 1992, 1993). Thus, normal growth does not necessarily indicate normal splicing.

Based on protein sequence comparisons and a prominent amino-terminal RS domain, the gene YCL11C (identified from yeast genomic sequencing) was suggested by Krainer and colleagues to be $S$. cerevisiae's U2AF65 (Birney et al. 1992). To test this prediction, we generated a deletion of the YCL11C ORF; the gene is inessential and extracts are wild type for complex formation and splicing (also see Baudin et al. 1993). More importantly, the gene deletion is viable in combination with the MUD2 KO gene, and extracts from the double mutant strain show no additional defects due to the YCL11C KO mutation (data not shown). As a consequence, we have no evidence that the gene product of YCL11C is involved in splicing.

An alternative possibility is that MUD2P and U2AF65 have a common ancestor, but MUD2P became less important (inessential) in parallel with other changes in $S$. cerevisiae pre-mRNA splicing. Perhaps the changes in $S$. cerevisiae splicing were also accompanied by changes in the functional role of the amino-terminal two-thirds of the protein. In this context, it may be relevant that $S$. pombe's pre-mRNA splicing not only employs an essential and well-conserved U2AF65 gene but also is generally more mammalian-like, for example, more introns and substantial polypyrimidine-rich regions (Rymond and Rosbash 1992).

The sequence conservation in the carboxy-terminal region of MUD2P and U2AF65 is striking. This third RBD has a number of atypical features, and these are also present in MUD2P (Krainer and Birney, pers. comm.; note the unusual but quite conserved RNP1 and RNP2 motifs). The resemblance presumably reflects a related target RNA sequence if not a similar functional role shared by this region of all four proteins. Based on this selective conservation, the different RBDs of U2AF65 might have different RNA targets, for example, the poly- 
pyrimidine stretch might be only one of two or more RNA motifs directly contacted by U2AF 65 .

MUD2P contacts pre-mRNA directly, for which the highly conserved yeast branchpoint as well as the integrity of its third RBD is required. It is therefore possible that the branchpoint sequence is directly contacted by this region of the protein. Although attractive, this hypothesis has not been confirmed by definitive identification of the pre-mRNA sequence that is cross-linked to MUD2P. As a consequence, it is possible that the branchpoint dependence is indirect and that the third RBD contacts some other sequence motif.

Overexpression of MUD2P does not lead to more MUD2P-pre-mRNA complex, indicating that the protein does not bind tightly and independently to the premRNA substrate under these conditions (Fig. 2B). Although this is at odds with the current view of U2AF function (avid binding to the pre-mRNA polypyrimidine stretch without additional proteins or factors; Zamore et al. 1992), the same experimental strategy has not been applied to both proteins. MUD2P binding to pre-mRNA has not been attempted with high concentrations of recombinant protein, and U2AF-pre-mRNA interactions have not been assayed with physiological protein concentrations in complete splicing extract. Under these conditions, it is possible that U2AF binding might manifest some U1 snRNP dependence. These possibilities underscore the uncertainty that U2AF and MUD2P bind before, with, or after U1 snRNP.

Although the interpretation of the experiments with PRP11 is complicated by the possibility that this protein might add during spliceosome assembly in an unexpected manner (Ruby et al. 1993), the most conservative interpretation is that PRP11 adds to the assembling spliceosome along with the rest of U2 snRNP. We favor this interpretation because (1) the biochemical characterization of PRP11 addition to splicing complexes (Fig. 7) recapitulates the ATP-dependent results obtained for PRP9 (Abovich et al. 1990) and PRP21 (Arenas and Abelson 19931; (2) studies in the yeast two-hybrid system have described interactions between PRP9, PRP21, and PRP11 and defined a tripartite complex (Legrain et al. 1993; Legrain and Chapon 1993); and (3) a single biochemical fraction from mammalian cells, SF3A, itself a subcomponent of U2 snRNP (Brosi et al. 1993), contains three proteins. One of these proteins $(60 \mathrm{~K})$ is the homolog of PRP9 (Brosi et al. 1993), and another (SAP62) is the homolog of PRP11 (Bennett and Reed 1993). The interaction between MUD2P and PRP11 therefore establishes a strong functional link between metazoan U2AF and MUD2P; both proteins aid U2 snRNP's addition to U1 commitment complexes. Because the interaction was assayed in yeast (raising the possibility that other yeast proteins bridge the contact), in vitro experiments with purified proteins will be required to verify that the interaction is direct. Taken together with the synthetic lethality, however, the data suggest that MUD2P makes direct contact with the PRP11 protein (Fig. 9C). The interaction is the first identified inter-snRNP protein-protein contact that bridges U1 snRNP and U2 snRNP dur-
A

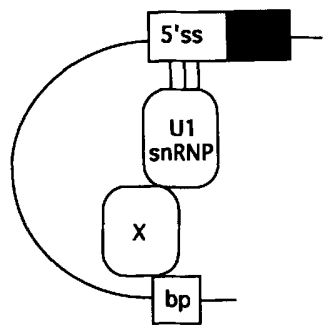

B

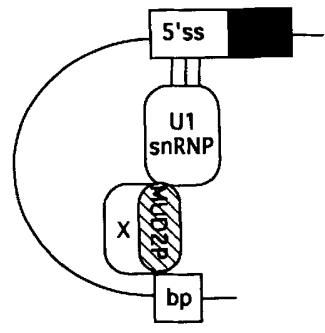

C

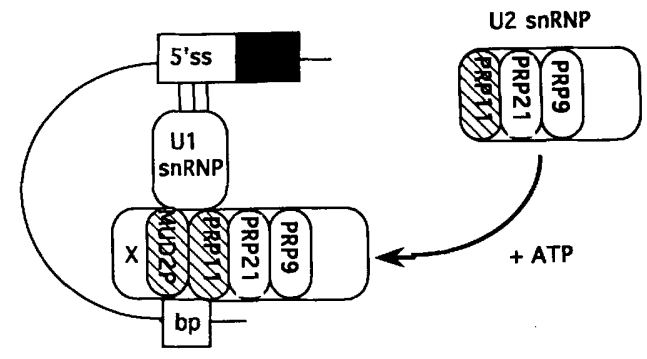

Figure 9. A model for early spliceosome assembly. $(A)$ In this model, U1 snRNP is base-paired to the $5^{\prime}$ splice site $\left(5^{\prime} \mathrm{SS}\right)$; the branchpoint region (bp) is shown interacting with Ul snRNP via an associated activity $X$ (Rosbash and Séraphin 1991). (B) We propose that MUD2P is a component of $X .(C)$ We further propose that MUD2P makes direct contact with the PRP11 protein during U2 snRNP recruitment to the complex.

ing spliceosome assembly. We have no comparable picture of the interactions that bridge the $5^{\prime}$ and $3^{\prime}$ splice site regions in the yeast commitment complex (Fig. 9B). In contrast, a network of interactions between several defined components is proposed to bridge the $5^{\prime}$ and $3^{\prime}$ splice sites in mammalian U1 snRNP complexes (Wu and Maniatis 1993).

The CC2 complex formed in the MUD2 KO extract is aberrant but still distinct from $\mathrm{CCl}$, the basal U1 snRNP complex (Fig. 1). It is therefore likely that there are other yeast commitment complex proteins in addition to MUD2P that are branchpoint dependent. Similar biochemical and genetic approaches should lead from MUD2 to these other genes. Their analysis should continue to illuminate the role of the branchpoint and branchpoint-dependent activities in early complex formation, and the role of early complex formation in yeast splice site identification. 


\section{Materials and methods \\ Cloning of the wild-type MUD2 gene and mapping of mud2 mutants}

The original mud2-1 mutant strain (Yml4) was used as the host for transformation with a wild-type yeast library based on the LEU2-CEN vector p366, as described (Liao et al. 1993). A functional 4.5-kb subclone (pXL79) was identified, and the boundaries of the MUD2 open reading frame mapped by linker insertion mutagenesis (Legrain et al. 1991). A portion (2.5 kb) of the sequence was determined (Liao 1992) and will be deposited in the gene bank. This sequence contains an open reading frame of 527 amino acids. The sequence of four mud2 alleles was determined as described previously for mud1 (Liao et al. 1993). All four mutations introduce stop codons within this open reading frame; mud2-1, mud2-2, and mud2-3 introduce stop codons after amino acids 448,183 , and 47 , respectively. mud2-4 contains a 1-nucleotide deletion at the position of amino acid 49 that frame-shifts the reading frame into a stop codon at position 73 .

\section{Plasmid constructions and strains}

To aid in subsequent manipulations, synthetic BamHI and SphI sites were introduced as unique sites flanking the $4.5-\mathrm{kb}$ insert of pXL79, and NcoI and SalI sites were introduced at the initiating ATG and 15 nucleotides downstream of the stop codon, respectively.

\section{Deletion of the MUD2 gene: MUD2 KO}

A plasmid was constructed in which the NcoI-Sal I fragment described above was replaced by a $2.2-\mathrm{kb}$ Sall-HpaI LEU2 fragment flanked by Sall and NcoI sites. Gene disruptions into wildtype yeast strain MGD 353-13D were carried out with the BamHI-SphI linear fragment of the resulting plasmid. Chromosomal deletions of MUD2 were confirmed by PCR amplification of genomic DNA from the transformants with appropriate primers or by Southern blotting.

\section{HA protein tagging}

Proteins were tagged with the HA epitope before the last amino acid at the carboxyl terminus as described previously (Abovich et al. 1990).

\section{MUD2 HA YCP}

The 4.5-kb BamHI-SphI fragment carrying the HA-tagged $M U D 2$ gene was cloned in YCP50 and introduced into the MUD2 KO strain.

\section{MUD2 HA Gal}

A synthetic BamHI site was placed in front of the NcoI site and an SstI site was introduced 550 nucleotides downstream of the stop codon in MUD2 HA to clone this fragment into BamHI-/ SstI-digested pLGSD5 (Guarente et al. 1982), and introduce it into the MUD2 KO strain. The expression of the MUD2HAP is driven by the Gal UAS promoter and is overexpressed when cells are grown in galactose. A very low level of expression persists when cells are grown in glucose.

\section{PRP11-HA Gal}

The PRP11-coding sequence was amplified by PCR from wildtype genomic DNA, with primers that included a BamHI site at the initiating methione and a XhoI site after the stop codon. Primers were designed from the PRP11 sequence (Chang et al. 1988). This amplified DNA was cloned in the above-described plasmid in place of the BamHI-SalI fragment coding for MUD2 HA. The resulting plasmid was transformed into strain A635 (Legrain and Rosbash 1989), a prp11 temperature-sensitive mutant. The gene was functional as verified by growing the transformed strain at $37^{\circ} \mathrm{C}$.

In vitro complex assembly and native electrophoresis

Splicing extracts were prepared, and standard splicing reactions were carried out as described previously (Abovich et al. 1990). Depletions of U2 snRNP and ATP were done as described in Liao et al. (Liao et al. 1992), and native gel electrophoresis was performed as in Séraphin and Rosbash (1989).

\section{Supershift of complexes}

After incubation for commitment complex formation, 12CA5 monoclonal antibody from BabCo (Berkely Antibody Company, Richmond, CA) was diluted in buffer D (Newman et al. 1985) and added to the reactions to a final concentration of $11.5 \mu \mathrm{g} / \mathrm{l}_{\text {; }}$ the control reactions received buffer D only. Samples were incubated at $25^{\circ} \mathrm{C}$ for $10 \mathrm{~min}$ prior to the addition of buffer $\mathrm{R}$ and nonspecific competitor, and subjected to electrophoresis as described previously (Séraphin and Rosbash 1989).

\section{UV cross-linking of commitment complexes}

Standard commitment complex reactions were scaled up sixfold, and the concentration of radiolabeled pre-mRNA increased fourfold. After incubation at $25^{\circ} \mathrm{C}$ for $30 \mathrm{~min}$, the reactions were transferred to a 96-well microtiter dish, placed on ice, and UV irradiated. After irradiation, the samples were treated with RNase A. Irradiation and RNase A digestion were done as described (Hoffman and Grabowski 1992).

\section{Immunoprecipitations}

Immunoprecipitations were carried out in NET-150 and washed at the same salt concentration (Abovich et al. 1990); immunoprecipitation of the cross-linked protein after UV and RNase A treatment were denatured prior to immunoprecipitation as recommended in Kolodziej and Young (1991). These immunoprecipitates were also washed at a higher salt concentration (NET$300)$.

\section{Analysis of protein-protein interactions in the yeast two-hybrid system}

We used the two-hybrid system to detect protein interactions by activation of transcription (Fields and Song 1989) as established and modified by Gyuris et al. (1993). The yeast host strain EGY48 and plasmids (pEG200 for LexA fusions; pJG4-5 for B42 activator fusions) and the reporter pSH18-34 were all a gift from Roger Brent's laboratory (Harvard Medical School, Boston, MA).

LexA-MUD2 fusion derivatives were generated by directional cloning into the BamHI and SalI sites in pEG200; and into the EcoRI and Sall sites for LexA-Per (Z. Huang, unpubl.).

B42-PRP11 and the variant prpll temperature-sensitive strain and PRP11-Cys were generated by directional cloning into the EcoRI and XhoI sites of pJG4-5, as was B42-Per (Z. Huang, pers. comm.). 


\section{Acknowledgments}

We thank Roger Brent's laboratory for their generous gift of the yeast strain and plasmids used in the two-hybrid system, and Zuoshi Huang for advice and the Drosophila per constructs used as controls. We also thank Hildur Colot and Jie Tang for providing the MUD13 and MUD1 KO strains, respectively. We are grateful to Hildur Colot, Claudio Pikielny, Francoise Stutz, Peter Hodges, Jean Beggs, Juan Valcarcel, Ravinder Singh, and Don Rio for their critical reading of this manuscript. We thank L.A. Monaghan for expert secretarial assistance. This work was supported by the National Institutes of Health grant GM23549.

The publication costs of this article were defrayed in part by payment of page charges. This article must therefore be hereby marked "advertisement" in accordance with 18 USC section 1734 solely to indicate this fact.

\section{References}

Abovich, N., P. Legrain, and M. Rosbash. 1990. The yeast PRP6 gene encodes a U4/U6 small nuclear ribonucleoprotein particle (snRNP) protein, and the PRP9 gene encodes a protein required for U2 snRNP binding. Mol. Cell. Biol. 10: 64176425.

Arenas, J.E. and J.N. Abelson. 1993. The Saccharomyces cerevisiae PRP21 gene product is an integral component of the prespliceosome. Proc. Natl. Acad. Sci. 90: 6771-6775.

Barabino, S., B.J. Blencowe, U. Ryder, B.S. Sproat, and A.I. Lamond. 1990. Targeted snRNP depletion reveals an additional role for mammalian U1 snRNP in spliceosome assembly. Cell 63: 293-302.

Baudin, A., O. Ozier-Kalogeropoulos, A. Denouel, F. Lacroute, and C. Cullin. 1993. A simple and efficient method for direct gene deletion in Saccharomyces cerevisiae. Nucleic Acids Res. 21: 3329-3330.

Bennett, M. and R. Reed. 1993. Correspondence between a mammalian spliceosome component and an essential yeast splicing factor. Science 262: 105-108.

Bennett, M., S. Michaud, J. Kingston, and R. Reed. 1992. Protein components specifically associated with prespliceosome and spliceosome complexes. Genes \& Dev. 6: 1986-2000.

Birney, E., S. Kumar, and A.R. Krainer. 1992. A putative homolog of $\mathrm{U}_{2} \mathrm{AF}^{65}$ in $S$. cerevisiae. Nucleic Acids Res. 20: 4663 .

Brosi, R., K. Groning, S.-E. Behrens, R. Luhrmann, and A. Kramer. 1993. Interaction of mammalian splicing factor SF3a with U2 snRNP and relationship of its $60-\mathrm{kD}$ subunit to yeast PRP9. Science 262: 102-105.

Caceres, J.R. and A.R. Krainer. 1993. Functional analysis of premRNA splicing factor SF2/ASF structural domains. EMBO $J$. 12: 4715-4726.

Chang, T-H., M.W. Clark, A.J. Lustig, M.E. Cusick, and J. Abelson. 1988. RNAll protein is associated with the yeast spliceosome and is localized in the periphery of the cell nucleus. Mol. Cell. Biol. 8: 2379-2393.

Fields, S. and O. Song. 1989. A novel genetic system to detect protein-protein interactions. Nature 340: 245-246.

Fu, X.-D. 1993. Specific commitment of different pre-mRNAs to splicing by single SR proteins. Nature 365: 82-85.

Green, M.R. 1991. Biochemical mechanisms of constitutive and regulated pre-mRNA splicing. Annu. Rev. Cell Biol. 7: 559599.

Guarente, L., R.R. Yocum, and P. Gifford. 1982. A GAL10-CYC1 hybrid yeast promoter identifies the $G A L 4$ regulatory region as an upstream site. Proc. Nat1. Acad. Sci. 79: 7410-7414.
Guthrie, C. 1991. Messenger RNA splicing in yeast: Clues to why the spliceosome is a ribonucleoprotein. Science 253: 157-163.

Gyuris, J., E. Golemis, H. Chertkov, and R. Brent. 1993. Cdil, a human $\mathrm{Gl}$ and $\mathrm{S}$ phase protein phosphatase that associates with Cdk2. Cell 75: 791-803.

Hoffman, B.E. and P.J. Grabowski. 1992. U1 snRNP targets an essential splicing factor, U2AF65, to the $3^{\prime}$ splice site by a network of interactions spanning the exon. Genes \& Dev. 6: $2554-2568$.

Huang, Z.J., I. Edery, and M. Rosbash. 1993. PAS is a dimerization domain common to Drosophila Period and several transcription factors. Nature 364: 259-262.

Jamison, S.F., A. Crow, and M.A. Garcia-Blanco. 1992. The spliceosome assembly pathway in mammalian extracts. Mol. Cell. Biol. 12: 4279-4287.

Kanaar, R., S.E. Roche, E.L. Beall, M.R. Green, and D.C. Rio. 1993. The conserved pre-mRNA splicing factor U2AF from Drosophila: Requirement for viability. Science 262: 569573.

Kolodziej, P.A. and R.A. Young. 1991. Epitope tagging and protein surveillance. Methods Enzymol. 194: 508-519.

Kunkel, T.A. 1985. Rapid and efficient site-specific mutagenesis without phenotypic selection. Proc. Natl. Acad. Sci. 82: 488-492.

Laemmli, U.K. 1970. Cleavage of structural proteins during the assembly of the head of bacteriophage T4. Nature 227: 680685.

Legrain, P. and C. Chapon. 1993. Interaction between PRP11 and SPP91 yeast splicing factors and characterization of a PRP9-PRP11-SPP91 complex. Science 262: 108-110.

Legrain, P. and M. Rosbash. 1989. Some cis- and trans-acting mutants for splicing target pre-mRNA to the cytoplasm. Cell 57: 573-583.

Legrain, P., B. Séraphin, and M. Rosbash. 1988. Early commitment of yeast pre-mRNA to the spliceosome pathway. Mol. Cell. Biol. 8: 3755-3760.

Legrain, P., C. Chapon, E. Schwob, R. Martin, M. Rosbash, and B. Dujon. 1991. Cloning of the two essential yeast genes, $P R P 6$ and PRP9, and their rapid mapping, disruption and partial sequencing using a linker insertion strategy. Mol. Gen. Genet. 225: 199-202.

Legrain, P., C. Chapon, and F. Galisson. 1993. Interactions between PRP9 and SPP91 splicing factors identify a protein complex required in prespliceosome assembly. Genes \& Dev. 7: 1390-1399.

Liao, X.C. 1992. "Pre-mRNA splicing in yeast: Roles of U1 snRNA and its associated protein." Ph.D. thesis, Brandeis University, Waltham, MA.

Liao, X.C., H.V. Colot, Y. Wang, and M. Rosbash. 1992. Requirements for U2 snRNP addition to yeast pre-mRNA. Nucleic Acids Res. 20: 4237-4245.

Liao, X.C., J. Tang, and M. Rosbash. 1993. An enhancer screen identifies a gene that encodes the yeast Ul snRNP A protein: Implications for snRNP protein function in pre-mRNA splicing. Genes \& Dev. 7: 419-428.

Lossky, M., G.J. Anderson, S.P. Jackson, and J. Beggs. 1987. Identification of a yeast snRNP protein and detection of snRNPsnRNP interactions. Cell 51: 1019-1026.

Michaud, S. and R. Reed. 1991. An ATP-independent complex commits pre-mRNA to the mammalian spliceosome assembly pathway. Genes \& Dev. 5: 2534-2546.

. 1993. A functional association between the $5^{\prime}$ and $3^{\prime}$ splice sites is established in the earliest prespliceosome complex (E) in mammals. Genes \& Dev. 7: 1008-1020.

Moore, M.J., C.C. Query, and P.A. Sharp. 1993. Splicing of pre- 
cursors to mRNAs by the spliceosome. In The RNA world (ed. R.F. Gesteland and J.F. Atkins), pp. 303-357. Cold Spring Harbor Laboratory Press, Cold Spring Harbor, New York.

Newman, A.J., R.-J. Lin, S. Cheng, and J. Abelson. 1985. Molecular consequences of specific intron mutations on yeast mRNA splicing in vivo and in vitro. Cell 42: 335-344.

Patterson, B. and C. Guthrie. 1991. A U-rich tract enhances usage of an alternative $3^{\prime}$ splice site in yeast. Cell 64: 181187.

Potashkin, J., K. Naik, and K. Wentz-Hunter. 1993. U2AF homolog required for splicing in vivo. Science 262: 573-575.

Rosbash, M. and B. Séraphin. 1991. Who's on first? The U1 snRNP-5' splice site interaction and splicing. Trends Biochem. Sci. 16: 187-190.

Ruby, S.W. and J. Abelson. 1988. An early hierarchic role of U1 small nuclear ribonucleoprotein in spliceosome assembly. Science 242: 1028-1035.

Ruby, S.W., T.-H. Chang, and J. Abelson. 1993. Four yeast spliceosomal proteins (PRP5, PRP9, PRP11, and PRP21) interact to promote U2 snRNP binding to pre-mRNA. Genes \& Dev. 7: 1909-1925.

Ruskin, B., P.D. Zamore, and M.R. Green. 1988. A factor, U2AF, is required for U2 snRNP binding and splicing complex assembly. Cell 52: 207-219.

Rymond, B.C. and M. Rosbash. 1985. Cleavage of 5' splice site and lariat formation are independent of 3 ' splice site in yeast mRNA splicing. Nature 317: 735-737.

1992. Yeast pre-mRNA splicing. In The molecular and cellular biology of the yeast Saccharomyces: Gene expression (ed. E.W. Jones, J.R. Pringle, and J.R. Broach), pp. 143192. Cold Spring Harbor Laboratory Press, Cold Spring Harbor, New York.

Schena, M. and K.R. Yamamoto. 1988. Mammalian glucocorticoid receptor derivatives enhance transcription in yeast. Science 241: 965-967.

Séraphin, B. and M. Rosbash. 1989. Identification of functional U1 snRNA-pre-mRNA complexes committed to spliceosome assembly and splicing. Cell 59: 349-358.

. 1991. The yeast branchpoint sequence is not required for the formation of a stable UI snRNP-pre-mRNA complex and is recognized in the absence of U2 snRNA. EMBO $I$. 10: 1209-1216.

Steitz, J.A. 1992. Splicing takes a Holliday. Science 257: 888889.

$\mathrm{Wu}, \mathrm{J}$. and T. Maniatis. 1993. Specific interactions between proteins implicated in splice site selection and regulated alternative splicing. Cell 75: 1061-1070.

Zamore, P.D. and M.R. Green. 1989. Identification, purification, and biochemical characterization of U2 small nuclear ribonucleoprotein auxiliary factor. Proc. Natl. Acad. Sci. 86: 9243-9247.

- 1991. Biochemical characterization of U2 snRNP auxiliary factor: an essential pre-mRNA splicing factor with a novel intranuclear distribution. EMBO /. 10: 207-214.

Zamore, P.D., J.G. Patton, and M.R. Green. 1992. Cloning and domain structure of the mammalian splicing factor U2AF. Nature 355: 609-614.

Zillmann, M., M.L. Zapp, and S.M. Berget. 1988. Gel electrophoretic isolation of splicing complexes containing Ul small nuclear ribonucleoprotein particles. Mol. Cell. Biol. 8: 814 821.

Zuo, P. and I.L. Manley. 1993. Functional domains of the human splicing factor ASF/SF2. EMBO $/$. 12: 4727-4737. 


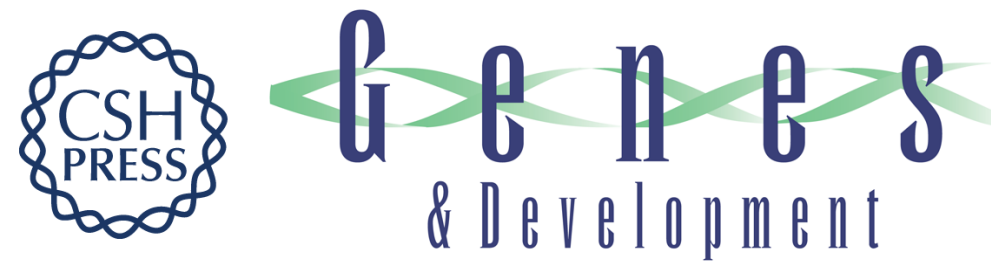

\section{The yeast MUD2 protein: an interaction with PRP11 defines a bridge between commitment complexes and U2 snRNP addition.}

N Abovich, X C Liao and M Rosbash

Genes Dev. 1994, 8:

Access the most recent version at doi:10.1101/gad.8.7.843

References This article cites 51 articles, 25 of which can be accessed free at:

http://genesdev.cshlp.org/content/8/7/843.full.html\#ref-list-1

License

Email Alerting

Service

Receive free email alerts when new articles cite this article - sign up in the box at the top right corner of the article or click here.

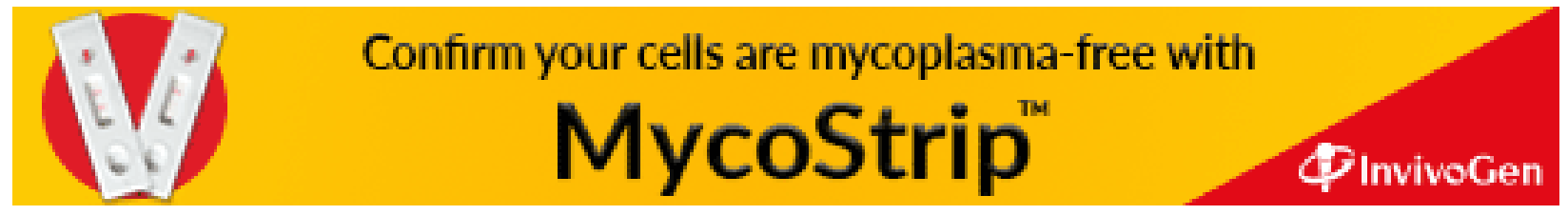

